\title{
Phonon assisted intersubband transitions in step quantum well structures
}

\author{
H. B. Teng, J. P. Sun, and G. I. Haddad \\ Department of Electrical Engineering and Computer Science, The University of Michigan, Ann Arbor, \\ Michigan 48109-2122 \\ Michael A. Stroscio \\ U.S. Army Research Office, P.O. Box 12211, Research Triangle Park, North Carolina 27709-2211
}

SeGi Yu and K. W. Kim

Department of Electrical and Computer Science, North Carolina State University, Raleigh, North Carolina 27695-7911

(Received 19 February 1998; accepted for publication 27 April 1998)

\begin{abstract}
We evaluate effects of heterointerfaces on optical phonon modes and phonon assisted electron intersubband transition rates in step quantum well structures for intersubband lasers. Various phonon modes and electron-phonon interaction Hamiltonians, including the interface modes, confined longitudinal-optical modes, and half space modes in the quantum well structures are calculated based on the macroscopic dielectric continuum model and microscopic analysis. The transfer matrix method is used to calculate the interface modes. The intersubband transition rates due to electron-phonon scattering by these phonon modes are evaluated using Fermi's golden rule, with the electron wave functions obtained by solving the Schrödinger equation for the heterostructures under investigation. Our results show that, compared with the transition rates in the same structures calculated using the bulk phonon modes and the bulk Fröhlich interaction Hamiltonian, the electron interface-phonon interactions give significantly larger transition rates up to an order of magnitude. Therefore, the effects of localized phonon modes, especially the interface modes, must be taken into consideration for optimal device design. (C) 1998 American Institute of Physics. [S0021-8979(98)03915-2]
\end{abstract}

\section{INTRODUCTION}

Optical phonon assisted electron transitions play an important role in novel intersubband lasers ${ }^{1-4}$ operating at midinfrared wavelengths since preferential intersubband transition rates are critical to establish and maintain the population inversion for the device operation. These lasers incorporate narrow quantum well regions that must have thicknesses as small as $30-50 \AA$. In these structures, the intersubband electron transitions induced by electron-phonon interactions are the dominant relaxation process when the intersubband separation between the lasing states is made close to one unit or a multiple of the bulk longitudinal-optical (LO) phonon energy.

It is well known that the shape and energies of optical phonon modes are modified by quantum wells. The presence of heterointerfaces gives rise to the confinement of LO phonons as well as localized phonons at the interfaces. ${ }^{5-9}$ Therefore, use of the bulk Fröhlich Hamiltonian may give inaccurate predictions of electron transition rates in such laser structures. The optical phonon confinement effects on electron transition rates were evaluated in Refs. 10 and 11.

In this work, we calculate the modified phonon modes, evaluate the intersubband transition rates due to interactions between electrons and these phonon modes, and compare them to the transition rates calculated using the bulk Fröhlich Hamiltonian. The structures studied are shown in Fig. 1, where materials 1,2 , and 3 are GaAs, $\mathrm{Al}_{0.25} \mathrm{Ga}_{0.75} \mathrm{As}$, and
$\mathrm{Al}_{0.4} \mathrm{Ga}_{0.6} \mathrm{As}$, respectively. The structure shown in Fig. 1(a) is asymmetrical, having three heterointerfaces, whereas Fig. 1(b) shows a symmetrical structure with four heterointerfaces. These step quantum well structures represent the quantum well laser cells where the phonon induced intersubband transitions are important. We will see that different structures give different optical phonon dispersion relations and different phonon electrostatic potential profiles. In Sec. II, the dispersion relations and electrostatic potentials of the optical phonon modes and electron-phonon interaction Hamiltonians for these two structures are obtained based on the dielectric continuum model and microscopic analysis; the interface phonon modes are calculated using the transfer matrix method. ${ }^{7}$ In Sec. III, we investigate the transition rates between the quasi-bound states in the step quantum well structures due to the interface and confined LO phonon modes. Comparisons are made to the rates predicted by the bulk Fröhlich interaction Hamiltonian. ${ }^{12-14}$ Fermi's golden rule is employed to calculate the transition rates. We found that in these structures the interface phonon scattering dominates the electron transitions and generally gives an order of magnitude larger transition rates than those by bulk modes. It is concluded that for preferential electron transition in intersubband laser structures the effects of the localized phonon modes, especially the interface phonon modes, must be included for optimal design of these structures. For example, to 


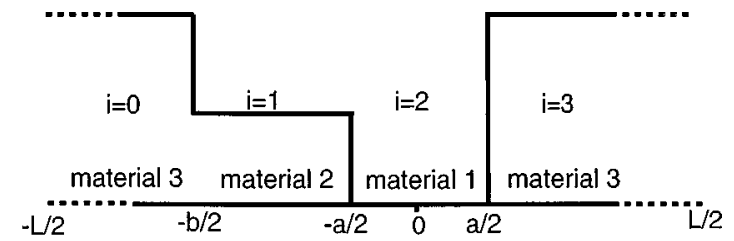

(a)

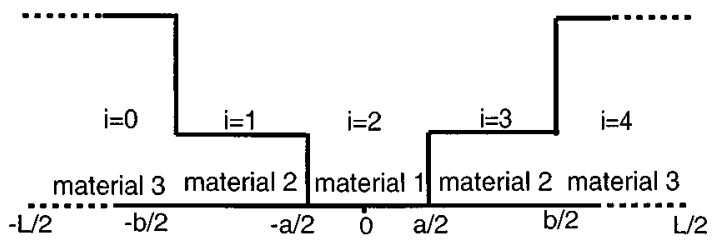

(b)

FIG. 1. Heterostructures used for the optical phonon mode calculations. Materials 1, 2, 3 are taken to be GaAs, $\mathrm{Al}_{0.25} \mathrm{Ga}_{0.75} \mathrm{As}$ and $\mathrm{Al}_{0.4} \mathrm{Ga}_{0.6} \mathrm{As}$, respectively.

facilitate electron transitions between two subbands, the subband energy separation should be set at the interface mode frequency.

\section{PHONONS IN QUANTUM WELL STRUCTURES}

Similar to electron confinement in the low-dimensional structures, both acoustic ${ }^{15}$ and optical phonons are modified in quantum well structures. In this work, we are mainly interested in the modified optical phonons because their energies are comparable to the desired intersubband separations. These phonon modes are the interface optical phonon modes, confined LO phonon modes, confined transverse-optical (TO) phonon modes, and half space LO and TO modes. While the confined and half space modes can be regarded as bulk modes "segmented" by the heterostructure interfaces, the interface modes are a new type of phonon with localized polarization and potential at the interfaces. These phonon modes can be calculated based on the dielectric continuum model and the microscopic analysis by Lucas, et al. ${ }^{5}$ and Licari and Evrard. ${ }^{6}$

We first obtain the functional form of the interface modes, the confined and half space LO modes, using the dielectric continuum model for the structure in Fig. 1(a). The electrostatic equations are given by

$$
\begin{aligned}
& \nabla \cdot \mathbf{D}(\mathbf{r})=\rho_{0}(\mathbf{r}), \\
& \mathbf{D}(\mathbf{r})=\epsilon \mathbf{E}(\mathbf{r})=\epsilon_{0} \mathbf{E}(\mathbf{r})+\mathbf{P}(\mathbf{r}), \\
& \mathbf{E}(\mathbf{r})=-\nabla \phi(\mathbf{r}),
\end{aligned}
$$

where $\mathbf{E}(\mathbf{r}), \mathbf{D}(\mathbf{r}), \mathbf{P}(\mathbf{r})$, and $\phi(\mathbf{r})$ are the electric field, electric displacement, electric polarization and scalar potential, $\epsilon$ and $\epsilon_{0}$ are dielectric constants of the respective materials and the permittivity of free space, respectively, and $\rho_{0}(\mathbf{r})$ is the free charge density. Considering free oscillation with free charge density $\rho_{0}(\mathbf{r})=0$, the above equations lead to

$$
\epsilon^{2} \phi(\mathbf{r})=0
$$

In a quantum well structure, since the system has no constraint on the $x-y$ plane, we can write

$$
\phi(\mathbf{r})=\frac{1}{\sqrt{A}} e^{i \mathbf{q} \cdot \rho} \phi(z),
$$

where $A$ is the in-plane cross section area of the structure, and $\mathbf{q}$ and $\rho$ are the two-dimensional in-plane wave vector and position vector, respectively. This gives

$$
\epsilon\left(\frac{\partial^{2}}{\partial z^{2}}-q^{2}\right) \phi(z)=0,
$$

where $q=|\mathbf{q}|$, the magnitude of the two-dimensional phonon in-plane wavevector. While Eq. (2) is general, Eq. (4) is applicable to two-dimensional quantum well systems. Applying Eq. (2) to bulk materials leads to the usual result of bulk LO phonons, with frequencies satisfying $\epsilon\left(\omega_{\mathrm{LO}}\right)=0$. The potential itself can be expanded in the Fourier form as the sum of the LO phonon modes with different wave vectors q. In a quantum well structure, Eq. (4) is satisfied by requiring either $\epsilon=0$ or $\left[\left(\partial^{2} / \partial z^{2}\right)-q^{2}\right] \phi(z)=0$. With $\epsilon=0, \phi$ can be any functions as long as the boundary conditions on $\mathbf{E}(\mathbf{r})$ and $\mathbf{D}(\mathbf{r})$ at the interfaces are satisfied.

For the asymmetrical structure shown in Fig. 1(a), Eq. (4) with $\epsilon=0$ leads to the following functional forms of the confined and half space LO modes. The frequencies of these modes are determined by setting $\epsilon=0$, and therefore are equal to the bulk LO mode frequencies $\omega_{L}$ of the respective materials. Although the materials in Fig. 1 are specific, the following results of the electrostatic potential can be easily extended to other ionic materials: for the confined phonon modes in region $i=2, \omega=\omega_{L 1}$ of material 1 ,

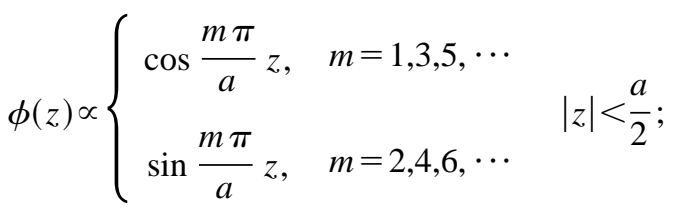

for the confined phonon modes in region $i=1, \omega=\omega_{L 2}$ of material 2,

$$
\begin{gathered}
\phi(z) \propto\left\{\begin{aligned}
\cos \frac{2 m \pi}{b-a}\left(z+\frac{b+a}{4}\right), & m=1,3,5, \cdots \\
\sin \frac{2 m \pi}{b-a}\left(z+\frac{b+a}{4}\right), & m=2,4,6, \cdots
\end{aligned}\right. \\
-\frac{b}{2}<z \leqslant-\frac{a}{2}
\end{gathered}
$$

for the half space LO modes in regions $i=0,3, \omega=\omega_{L 3}$ of material 3,

$$
\phi(z) \propto\left\{\begin{array}{c}
\sin \frac{2 \pi m}{L}\left(z+\frac{b}{2}\right), \quad m=1,2,3, \cdots \quad z \leqslant-\frac{b}{2}, \\
\sin \frac{2 \pi m}{L}\left(z-\frac{a}{2}\right), \quad m=1,2,3, \cdots \quad z \geqslant \frac{a}{2} .
\end{array}\right.
$$


TABLE I. Dielectric constants and phonon frequencies used in the calculation of the dispersion relations.

\begin{tabular}{lccc}
\hline \hline & $\mathrm{GaAs}$ & $\mathrm{AlAs}$ & $\mathrm{Al}_{x} \mathrm{Ga}_{1-x} \mathrm{As}$ \\
\hline$\epsilon(\infty)$ & 10.89 & 8.16 & $10.89-2.73 \times x$ \\
$\hbar \omega_{\mathrm{LA}}(\mathrm{meV})$ & 36.25 & $\cdots$ & $36.25-6.55 \times x+1.79 \times x^{2}$ \\
$\hbar \omega_{\mathrm{TA}}(\mathrm{meV})$ & 33.29 & $\cdots$ & $33.29-0.64 \times x-1.16 \times x^{2}$ \\
$\hbar \omega_{\mathrm{LB}}(\mathrm{meV})$ & $\cdots$ & 50.09 & $44.63+8.78 \times x-3.32 \times x^{2}$ \\
$\hbar \omega_{\mathrm{TB}}(\mathrm{meV})$ & $\cdots$ & 44.88 & $44.63+0.55 \times x-0.30 \times x^{2}$ \\
\hline \hline
\end{tabular}

The interface phonon modes can be obtained when $\epsilon$ $\neq 0$, then the solution for $\left[\left(\partial^{2} / \partial z^{2}\right)-q^{2}\right] \phi(z)=0$ is a linear combination of exponential terms, i.e.,

$$
\phi_{i}(q, z)=c_{i-} e^{-q z}+c_{i+} e^{+q z},
$$

where $i=0-3$ denotes the four regions in Fig. 1(a), and $c_{i \pm}$ 's are coefficients to be determined. The boundary conditions are

$$
\begin{aligned}
& \phi_{1}(q,-L / 2)=0, \\
& \phi_{i}\left(q, z_{i}\right)=\phi_{i-1}\left(q, z_{i}\right), \\
& \epsilon_{i} \frac{\partial}{\partial z} \phi_{i}\left(q, z_{i}\right)=\epsilon_{i-1} \frac{\partial}{\partial z} \phi_{i-1}\left(q, z_{i}\right), \\
& \phi_{3}(q, L / 2)=0,
\end{aligned}
$$

where $z_{i}$ is the location of the $i$ th interface: $z_{1}=-b / 2, z_{2}$ $=-a / 2$, and $z_{3}=a / 2$. With these boundary conditions, the coefficients are determined up to a constant. From Eq. (9) we readily find $c_{0-}=0$. With Eqs. (10) and (11) all other coefficients can be recursively expressed as functions of $\epsilon$ 's which are proportional to $c_{0+}$. The coefficient $c_{0+}$ can be obtained from orthonormality and completeness conditions to be discussed shortly. Since the boundary condition (12) requires $c_{3}$ to be zero, the following equation determines the dispersion relations for the interface modes:

$$
\begin{aligned}
& \frac{1}{\epsilon_{m 1} \epsilon_{m 2} \epsilon_{m 3}}\left[\left(\epsilon_{m 3}-\epsilon_{m 1}\right)\left(\epsilon_{m 1}+\epsilon_{m 2}\right)\left(\epsilon_{m 2}-\epsilon_{m 3}\right) e^{-q(a+b)}\right. \\
& \quad+\left(\epsilon_{m 3}-\epsilon_{m 1}\right)\left(\epsilon_{m 1}-\epsilon_{m 2}\right)\left(\epsilon_{m 2}+\epsilon_{m 3}\right) e^{-2 q a} \\
& \quad+\left(\epsilon_{m 3}+\epsilon_{m 1}\right)\left(\epsilon_{m 1}-\epsilon_{m 2}\right)\left(\epsilon_{m 2}-\epsilon_{m 3}\right) e^{-q(b-a)} \\
& \left.\quad+\left(\epsilon_{m 3}+\epsilon_{m 1}\right)\left(\epsilon_{m 1}+\epsilon_{m 2}\right)\left(\epsilon_{m 2}+\epsilon_{m 3}\right)\right]=0
\end{aligned}
$$

where $\epsilon_{m n}(\omega)$ is the dielectric constant of material $n$. While the normal relation $\epsilon_{m n}(\omega)=\epsilon_{m n}(\infty)\left(\omega^{2}-\omega_{\mathrm{Ln}}^{2}\right) /\left(\omega^{2}-\omega_{\mathrm{Tn}}^{2}\right)$ can be used for the dielectric function of binary compounds, appropriate expressions for ternary and even more complex systems can be found in Ref. 16. For the material shown in Fig. 1, we use ${ }^{7}$

$$
\epsilon_{m n}(\omega)=\epsilon_{m n}(\infty) \frac{\left(\omega^{2}-\omega_{\mathrm{LAn}}^{2}\right)\left(\omega^{2}-\omega_{\mathrm{LBn}}^{2}\right)}{\left(\omega^{2}-\omega_{\mathrm{TAn}}^{2}\right)\left(\omega^{2}-\omega_{\mathrm{TBn}}^{2}\right)},
$$

where values for the dielectric constants and the phonon frequencies of GaAs, AlAs, and $\mathrm{Al}_{x} \mathrm{Ga}_{1-x} \mathrm{As}$ are listed in Table I.

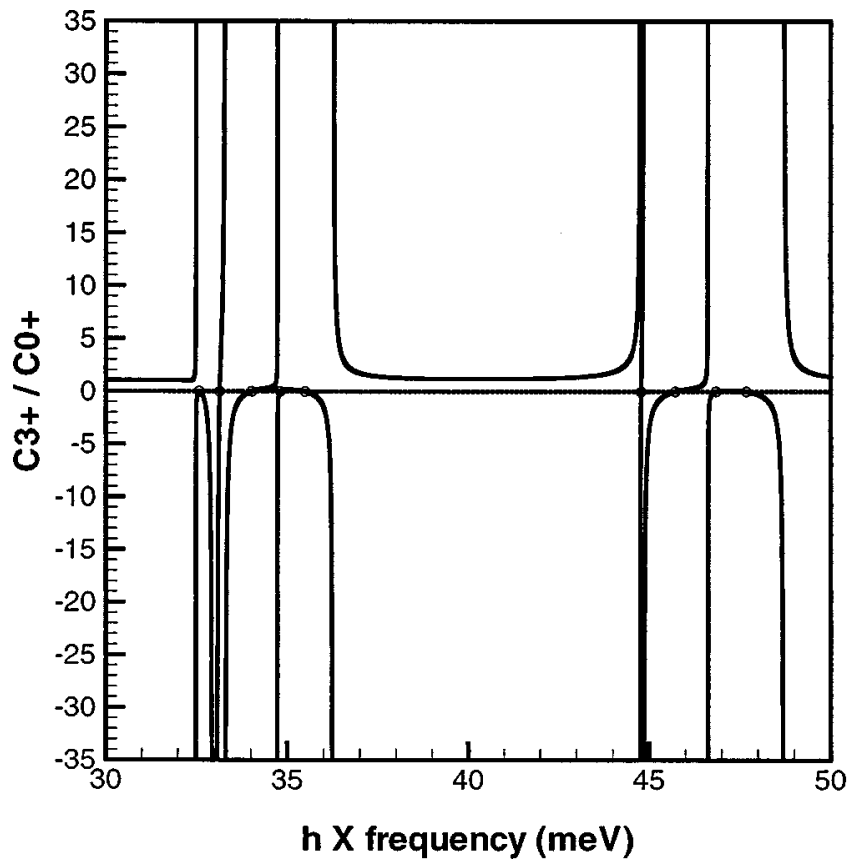

FIG. 2. Solutions to $c_{3+}(\omega)=0$ which give ten eigenfrequencies of the interface modes. For this plot, $q a=3$. Other parameters are given in Table I. The dispersion relations for these ten modes are shown in Fig. 3. Note that the lowest two eigenfrequencies are close to each other.

With these expressions and parameters, the coefficient $c_{3+}$ is plotted in Fig. 2 as a function of frequency for $q a$ $=3$. At the characteristic frequencies $\omega_{\mathrm{LAn}}, \omega_{\mathrm{LBn}}, \omega_{\mathrm{TAn}}$, and $\omega_{\mathrm{TBn}}$, the coefficient $c_{3}$ jumps between $\pm \infty$. The frequencies at which $c_{3+}$ vanishes give the eigenfrequencies of the interface modes. From Fig. 2 we see that equation $c_{3+}$ $=0$ has ten solutions, corresponding to the ten interface modes in the structure of Fig. 1(a). Equation (13) is numerically solved for different $q$ in order to obtain dispersion relations for these ten modes. The result is plotted in Fig. 3, with six GaAs-like modes having energies from 32 to 37 $\mathrm{meV}$, and four AlAs-like around $46 \mathrm{meV}$.

The macroscopic dielectric continuum model based on classical electrostatics involves relatively simple formulations. It gives the functional form of the interface modes, confined and half space LO modes, but it cannot give TO modes because TO modes do not produce macroscopic electric field and charge density. Fortunately the TO modes do not interact with electrons for the same reason and we can ignore them completely. However, to formulate the interaction Hamiltonians between electrons and the interface, confined and half space LO phonon modes, we also need the amplitudes of these modes. These amplitudes can be derived from the orthonormality and completeness conditions of the phonon eigenfunctions, which can only be formulated in the microscopic framework. Assuming the standard forms for the continuum expressions of the ionic force equation and the polarization of the polar medium, the normalization relation is derived from an appropriate generalization of the optical-phonon normalization condition and is given by ${ }^{7}$ 


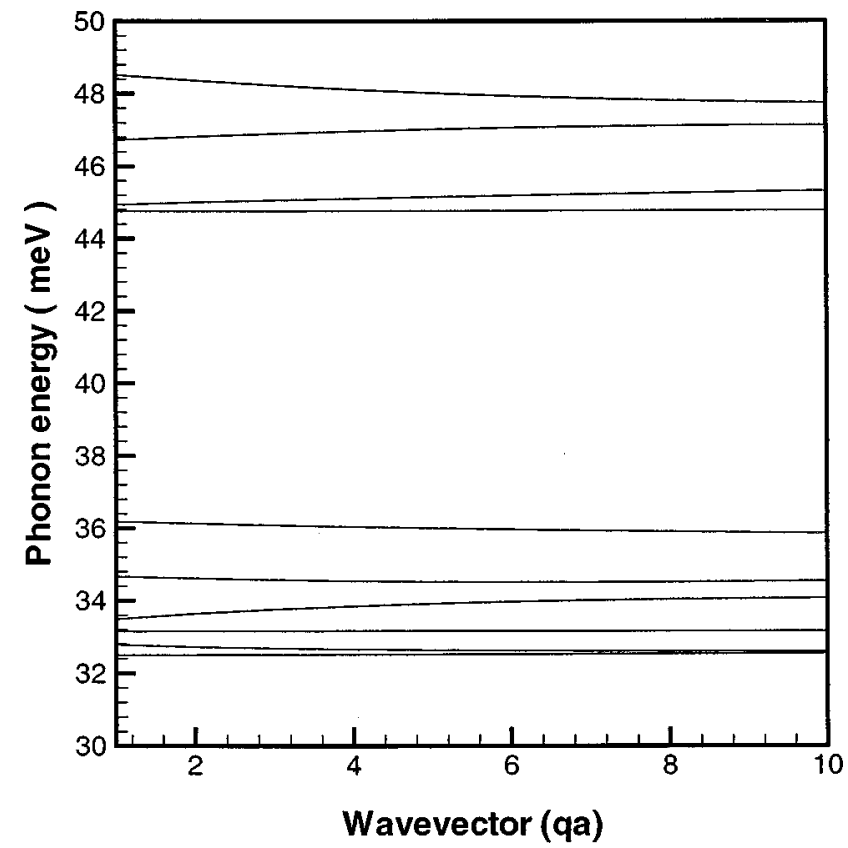

FIG. 3. Dispersion for the interface modes in structure 1(a). Ten interface modes are shown, with six GaAs-like modes from 32 to $36 \mathrm{meV}$, and four AlAs-like around $47 \mathrm{meV}$. Note that the lowest two curves are close to each other.

$$
\begin{aligned}
\frac{\hbar}{2 \omega}= & \sum_{i} \frac{\epsilon_{0}}{2 \omega} \frac{\partial \epsilon_{i}(\omega)}{\partial \omega} \int_{R_{i}} d z\left(q^{2}\left|\phi_{i}(q, z)\right|^{2}\right. \\
& \left.+\left|\frac{\partial \phi_{i}(q, z)}{\partial z}\right|^{2}\right),
\end{aligned}
$$

from which the amplitudes of electric potential for different modes can be determined. For the confined LO and half space modes in structure 1(a), we have the following results of the electrostatic potential:

for the confined phonon modes in region $2\left(\omega=\omega_{L 1}\right)$,

$$
\begin{aligned}
\phi(z)= & \left(\frac{\hbar}{\epsilon_{0}} \frac{1}{\partial \epsilon_{i}(w) / \partial w}\right)^{1 / 2}\left(\frac{1}{q^{2}+\left(\frac{m \pi}{a}\right)^{2}}\right)^{1 / 2}\left(\frac{2}{a}\right)^{1 / 2} \\
& \times \begin{cases}\cos \frac{m \pi}{a} z, & m=1,3,5, \cdots \\
\sin \frac{m \pi}{a} z, & m=2,4,6, \cdots\end{cases}
\end{aligned}
$$

for the confined phonon modes in region $1\left(\omega=\omega_{L 2}\right)$,

$$
\begin{aligned}
\phi(z)= & \left.\frac{\hbar}{\epsilon_{0}} \frac{1}{\partial \epsilon_{i}(w) / \partial w}\right)^{1 / 2}\left(\frac{1}{q^{2}+\left(\frac{2 m \pi}{b-a}\right)^{2}}\right)^{1 / 2}\left(\frac{4}{b-a}\right)^{1 / 2} \\
& \times\left\{\begin{array}{l}
\cos \frac{2 m \pi}{b-a}\left(z+\frac{b+a}{4}\right), \quad m=1,3,5, \cdots \\
\sin \frac{2 m \pi}{b-a}\left(z+\frac{b+a}{4}\right), \quad m=2,4,6, \cdots
\end{array}\right. \\
& -\frac{b}{2}<z \leqslant-\frac{a}{2} ;
\end{aligned}
$$

and for the half space LO modes in regions 0 and $3(\omega$ $\left.=\omega_{L 3}\right)$,

$$
\begin{aligned}
\phi(z)= & \left(\frac{\hbar}{\epsilon_{0}} \frac{1}{\partial \epsilon_{i}(w) / \partial w}\right)^{1 / 2}\left(\frac{1}{q^{2}+\left(\frac{2 \pi m}{L}\right)^{2}}\right)^{1 / 2}\left(\frac{4}{L}\right)^{1 / 2} \\
& \times\left\{\begin{array}{l}
\sin \frac{2 \pi m}{L}\left(z+\frac{b}{2}\right), \quad m=1,2,3, \cdots \quad z \leqslant-\frac{b}{2}, \\
\sin \frac{2 \pi m}{L}\left(z-\frac{a}{2}\right), \quad m=1,2,3, \cdots \quad z \geqslant \frac{a}{2} .
\end{array}\right.
\end{aligned}
$$

Expressions for the interface modes are also obtained. Instead of giving the long expressions for the coefficients $c_{i \pm}$, we plot some typical interface modes in Fig. 4, where also included are the confined LO modes and half space modes with $m=1,2$. As can be seen in Fig. 4, the interface modes are localized at the interfaces, whereas the confined and half space modes are sinusoidal. For the interface modes, the amplitude is typically concentrated at the interface, where the dielectric constant changes sign and has large absolute values on both sides.

The procedure for the interface mode calculation is formulated in a matrix form and referred to as the transfer matrix method. ${ }^{7}$

For structure 1(b), the dispersion relations of the interface modes are given by $c_{4+} / c_{0+}=0$, with $c_{4+}$ obtained from the same iteration procedure used for structure 1(a):

$$
\begin{aligned}
& \frac{1}{\epsilon_{m 1} \epsilon_{m 2}^{2} \epsilon_{m 3}}\left[-\left(\epsilon_{m 3}-\epsilon_{m 2}\right)^{2}\left(\epsilon_{m 2}+\epsilon_{m 1}\right)^{2} e^{2 q b}\right. \\
& \quad-2\left(\epsilon_{m 3}^{2}-\epsilon_{m 2}^{2}\right)\left(\epsilon_{m 2}^{2}-\epsilon_{m 1}^{2}\right) e^{q(a+b)} \\
& \quad+\left(\epsilon_{m 3}-\epsilon_{m 2}\right)^{2}\left(\epsilon_{m 2}-\epsilon_{m 1}\right)^{2} e^{2 q(b-a)}+2\left(\epsilon_{m 3}^{2}-\epsilon_{m 2}^{2}\right) \\
& \quad \times\left(\epsilon_{m 2}^{2}-\epsilon_{m 1}^{2}\right) e^{q(b-a)}-\left(\epsilon_{m 3}+\epsilon_{m 2}\right)^{2}\left(\epsilon_{m 2}-\epsilon_{m 1}\right)^{2} e^{2 q a} \\
& \left.\quad+\left(\epsilon_{m 3}+\epsilon_{m 2}\right)^{2}\left(\epsilon_{m 2}+\epsilon_{m 1}\right)^{2}\right]=0 .
\end{aligned}
$$

This equation has 14 solutions as shown in Fig. 5, corresponding to six GaAs-like and eight AlAs-like interface modes. The potential of some typical interface modes for this structure are plotted in Fig. 6. Due to the symmetry of this structure, they can be categorized as symmetric interface modes $[\phi(z)=\phi(-z)]$ and antisymmetric interface modes $[\phi(z)=-\phi(-z)]$.

The electrostatic potential of the confined and half space phonon modes for this structure are:

for the confined phonon modes in region $2\left(\omega=\omega_{L 1}\right)$, 

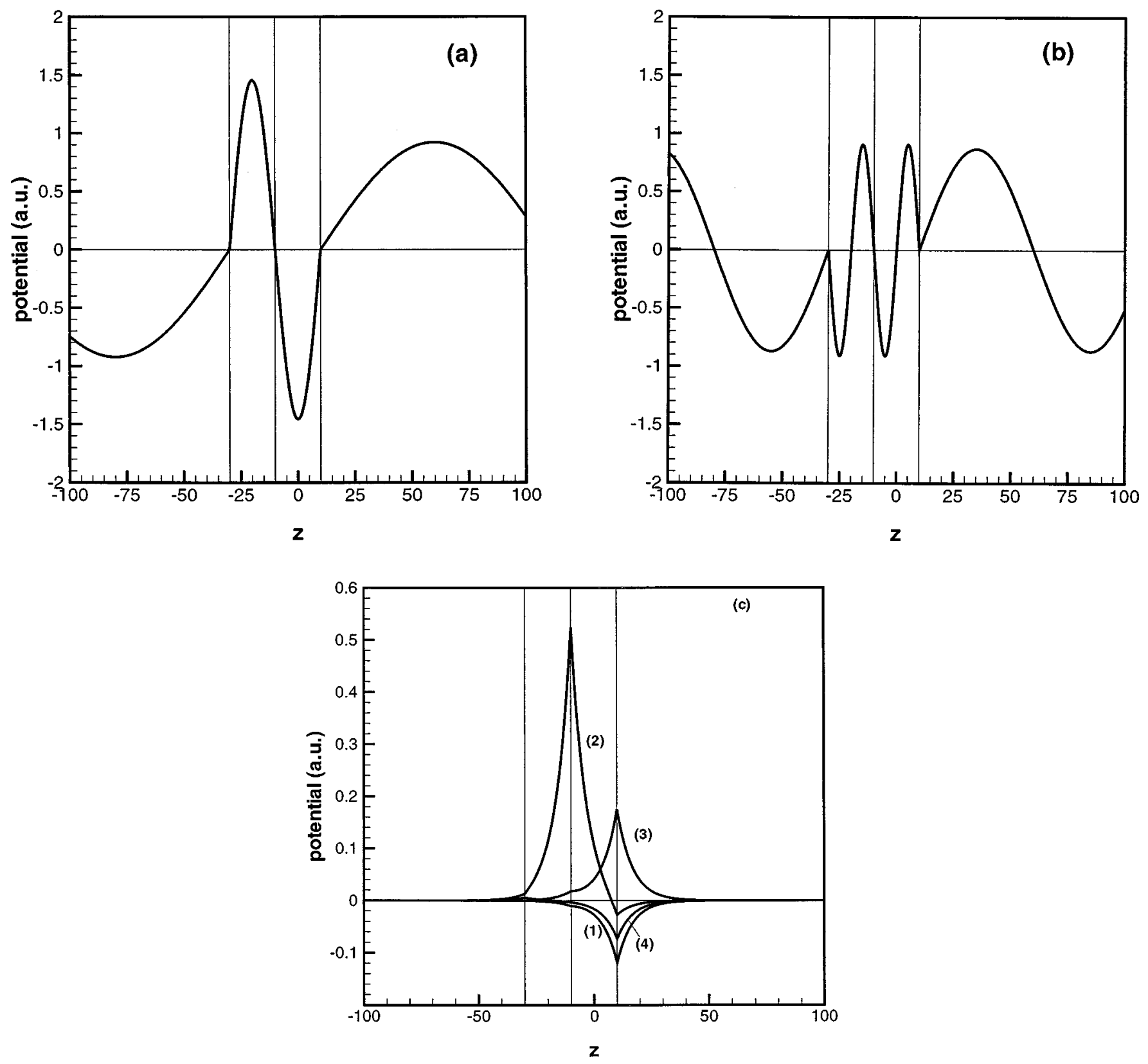

FIG. 4. Electrostatic potential for the phonon modes in structure 1(a): the half space modes in region 1, confined LO mode in region 2, confined LO mode in region 3, and half space mode in region 4 for (a) $m=1$ and (b) $m=2$. Four typical interface modes are shown in (c), with energies equal to (1) 46.84 meV, (2) $35.48 \mathrm{meV}$, (3) $34.81 \mathrm{meV}$, and (4) $32.57 \mathrm{meV}$, respectively. Vertical lines represent heterointerfaces. For this plot, $q a=3$.

$$
\begin{aligned}
\phi(z)= & \left(\frac{\hbar}{\epsilon_{0}} \frac{1}{\partial \epsilon_{i}(w) / \partial w}\right)^{1 / 2}\left(\frac{1}{q^{2}+\left(\frac{m \pi}{a}\right)^{2}}\right)^{1 / 2}\left(\frac{2}{a}\right)^{1 / 2} \\
& \times \begin{cases}\cos \frac{m \pi}{a} z, & m=1,3,5, \cdots \\
\sin \frac{m \pi}{a} z ; & m=2,4,6, \cdots\end{cases} \\
& |z|<\frac{a}{2} ;
\end{aligned}
$$

for the confined phonon modes in regions 1 and $3(\omega$ $\left.=\omega_{L 2}\right)$,

$$
\begin{aligned}
\phi(z)= & \left(\frac{\hbar}{\epsilon_{0}} \frac{1}{\partial \epsilon_{i}(w) / \partial w}\right)^{1 / 2}\left(\frac{1}{q^{2}+\left(\frac{2 m \pi}{b-a}\right)^{2}}\right)^{1 / 2}\left(\frac{4}{b-a}\right)^{1 / 2} \\
& \times\left\{\begin{array}{l}
\cos \frac{2 m \pi}{b-a}\left(z \pm \frac{b+a}{4}\right), \quad m=1,3,5, \cdots \\
\sin \frac{2 m \pi}{b-a}\left(z \pm \frac{b+a}{4}\right), \quad m=2,4,6, \cdots
\end{array}\right.
\end{aligned}
$$

where the plus sign is for $(b / 2)<z \leqslant-(a / 2)$, and the minus sign for $b / 2>z \geqslant a / 2$; and for the half space LO modes in regions 0 and $4\left(\omega=\omega_{L 3}\right)$, 


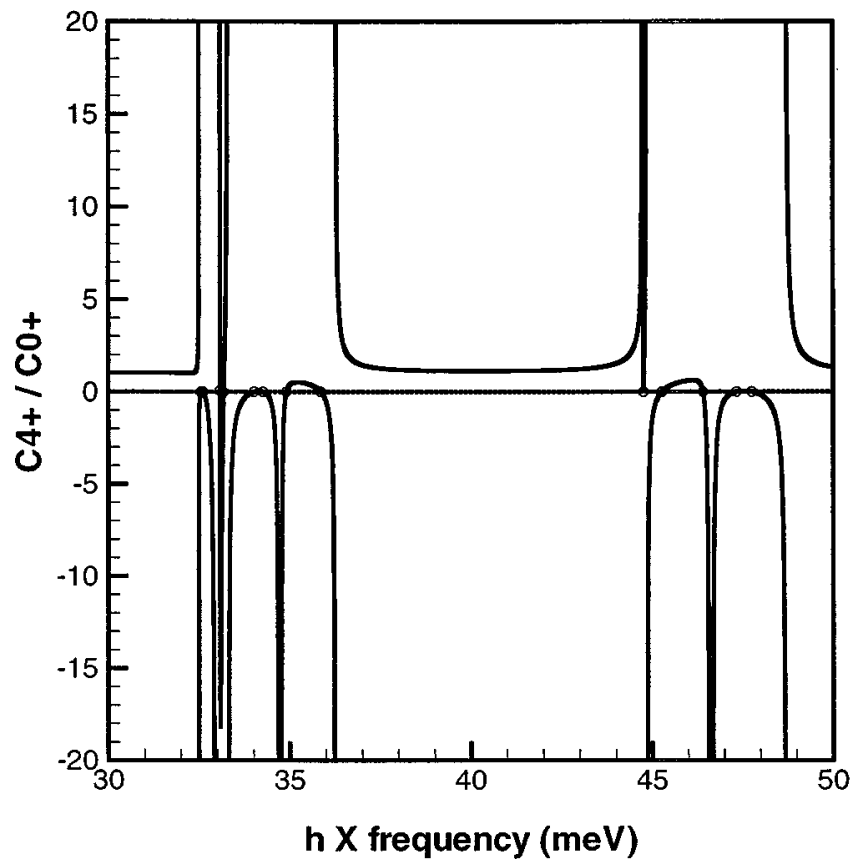

FIG. 5. Solutions to $c_{4+}(\omega)=0$ for the interface modes in structure 1(b), which give 14 eigenfrequencies of the interface modes. For this plot, $q a=0.5$. Other parameters are given in Table I. Note that the lowest two GaAs-like solutions are very close to each other.

$$
\begin{aligned}
\phi(z)= & \left(\frac{\hbar}{\epsilon_{0}} \frac{1}{\partial \epsilon_{i}(w) / \partial w}\right)^{1 / 2}\left(\frac{1}{q^{2}+\left(\frac{2 \pi m}{L}\right)^{2}}\right)^{1 / 2}\left(\frac{4}{L}\right)^{1 / 2} \\
& \times \sin \frac{2 \pi m}{L}\left(z \pm \frac{b}{2}\right), \quad m=1,2,3, \cdots
\end{aligned}
$$

where the plus sign is for $z \leqslant-(b / 2)$, and the minus sign for $z \geqslant b / 2$.

There are other forms of solutions which may not be categorized as the confined, half space, or interface modes. These modes are similar to the interface modes in some regions and to the confined or half space modes in other regions. For example, there are the modes which are half spacelike in regions 0 and 3 , but are interfacelike in regions 1 and 2 for structure 1(a). The contribution to electron transition by these modes is either interface modelike or confined modelike, depending on whether they are interface modelike or confined modelike in the most active region of the device. We will refer to both the interface modes and the modes which are interface modelike in the active region as "surface modes."

The interaction between electrons and a particular phonon mode $s$ is given by the Hamiltonian

$$
H_{e-\mathrm{ph}}=-e \sum_{q} \frac{1}{\sqrt{A}} e^{i \mathbf{q} \cdot \rho} \phi_{s}(z)\left(a_{-\mathbf{q}}^{\dagger}+a_{\mathbf{q}}\right),
$$

where $(1 / \sqrt{A}) e^{i \mathbf{q} \cdot \rho} \phi_{s}(z)$ is the electrostatic potential function for the phonon mode $s$, with in-plane wave vector $\mathbf{q}$, and $a_{\mathbf{q}}^{\dagger}\left(a_{\mathbf{q}}\right)$ is the creation (annihilation) operator of phonons.

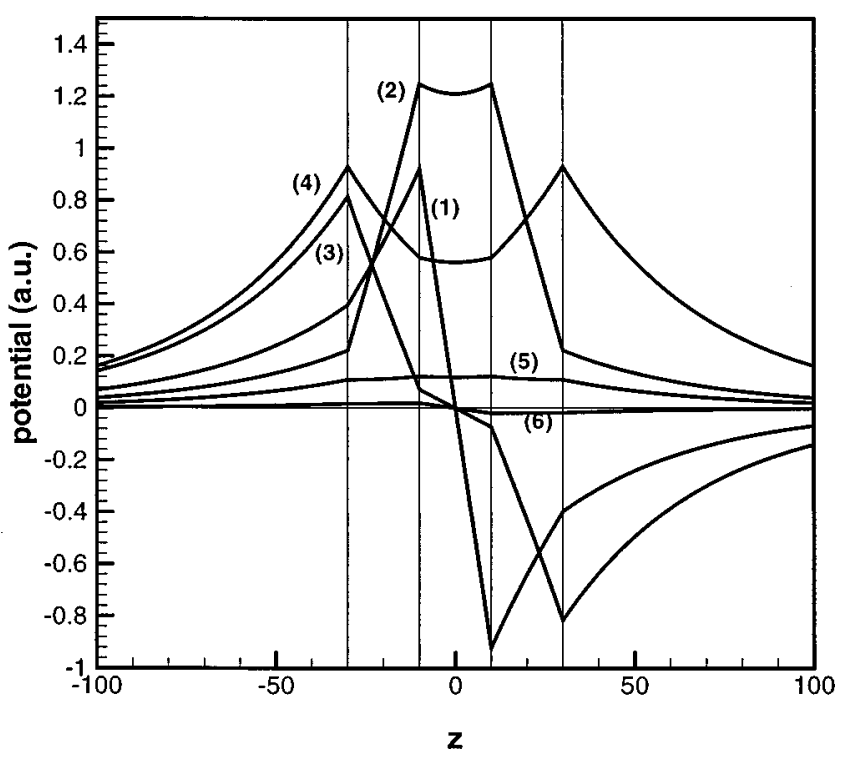

FIG. 6. Electrostatic potential for some typical interface phonon modes in structure 1(b). Due to the symmetry of the structure, the modes are either symmetric or antisymmetric. The frequencies of these modes are (1)35.86 meV, (2)34.91 meV, (3)34.26 meV, (4)34.01 meV, (5)33.17 meV, (6)33.07 $\mathrm{meV}$, respectively. Vertical lines represent heterointerfaces. For this plot, $q a=0.5$. Other parameters are given in Table $\mathrm{I}$.

\section{PHONON-ASSISTED INTERSUBBAND TRANSITIONS}

Neglecting the nearly flat $\mathbf{q}$ dependence on phonon energy (see Fig. 3 for an example of the interface mode dispersion), the transition processes can be visualized in Fig. 7. For emission, an electron in the upper subband with in-plane wave vector $\mathbf{k}$ can emit a phonon and jump to one of the accessible final states in the lower subband (shown as the equal-energy circle in the lower subband). For absorption, an electron in the lower band absorbs a phonon and jumps to one of the states on the circle in the upper band. The phonon induced transition rate of a single transition event between two electronic states $|n, \mathbf{k}\rangle$ and $\left|n^{\prime}, \mathbf{k}^{\prime}\right\rangle$ is given by Fermi's golden rule:

$$
\begin{aligned}
W_{n, n^{\prime}}^{\left\{_{e}{ }^{a_{e}}\right\}}\left(\mathbf{k}, \mathbf{k}^{\prime}\right)= & \frac{2 \pi}{\hbar}\left|\left\langle n^{\prime} \mathbf{k}^{\prime}, N_{q} \pm 1\left|H_{e-\mathrm{ph}}\right| n \mathbf{k}, N_{q}\right\rangle\right|^{2} \\
& \times \delta\left(E_{n^{\prime}}+E_{\mathbf{k}^{\prime}} \pm \hbar \omega-E_{n}-E_{k}\right),
\end{aligned}
$$

where $E_{n}$ and $E_{n^{\prime}}$ are the band-edge energies of the $n$th and $n^{\prime}$ th subbands, $\mathbf{k}$ and $\mathbf{k}^{\prime}$ are the in-plane electron wave vectors of the initial and final states, respectively, $E_{\mathbf{k}}$ $=\hbar^{2} k^{2} / 2 m$ is the in-plane energy, $\omega$ is the frequency of a given phonon mode, and $N_{q}$ is the phonon population based on the Bose-Einstein statistics. In addition, the superscripts $e$ and $a$ denote emission process and absorption process, respectively. The emission and absorption rates are generally of the same order and have similar in-plane energy dependence. For given states $|n, \mathbf{k}\rangle,\left|n^{\prime}, \mathbf{k}^{\prime}\right\rangle$ and a given phonon mode, the ratio of the emission and absorption rates, $W_{n, n^{\prime}}^{e}\left(\mathbf{k}, \mathbf{k}^{\prime}\right) / W_{n, n^{\prime}}^{a}\left(\mathbf{k}, \mathbf{k}^{\prime}\right)=\left(N_{q}+1\right) / N_{q}=e^{\hbar \omega / k_{B} T}$, is constant at a specified temperature. The difference between these two processes lies in whether the emission or absorption process is forbidden or requires an onset in-plane energy 

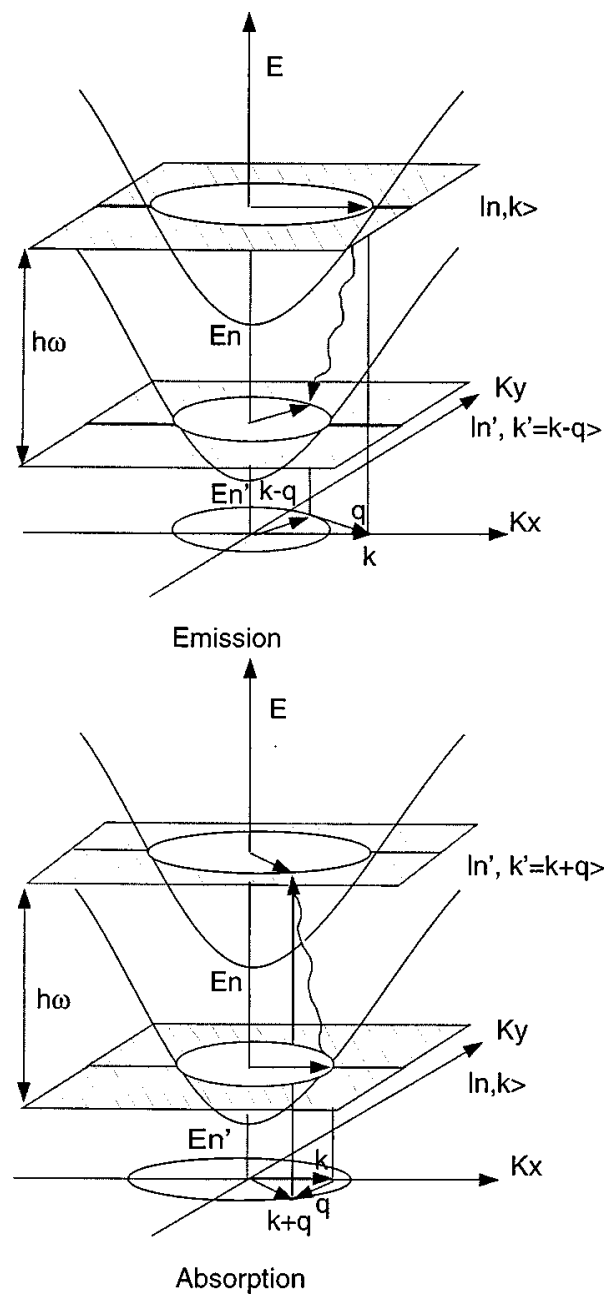

FIG. 7. Electron emission and absorption between initial state $|n, \mathbf{k}\rangle$ and final state $\left|n^{\prime}, \mathbf{k}^{\prime}\right\rangle$. For emission, the accessible final states are those on the circle in the lower subband. For absorption, the accessible final states are those in the upper subband.

required by energy conservation. When the subband separation is smaller than the phonon energy, an electron emitting a phonon has to possess a certain amount of in-plane energy $E_{\mathbf{k}}$ for the transition to occur. For phonon absorption, the transition can always happen when $E_{n^{\prime}}-E_{n} \leqslant \hbar \omega$. If the subband separation is larger than the phonon energy, then the emission can always happen while the absorption requires the electron to possess a certain amount of initial in-plane energy. We will hereafter focus only on the emission process and drop the superscripts of $W$, while indicating the difference in absorption case when appropriate.

The electron wave function can be written as

$$
|n, \mathbf{k}\rangle=\frac{1}{\sqrt{A}} e^{i \mathbf{k} \cdot \rho} \psi_{n}(z) .
$$

In writing $\psi_{n}$ independent of $k$, we have neglected the effect of kinetic energy confinement. $\psi_{n}(z)$ in the quantum well structures are obtained numerically by solving the Schrödinger equation. The structures and the wave functions of the first two levels in each structure are plotted in Figs. 8 and 9, respectively. As in the interface mode calculation described

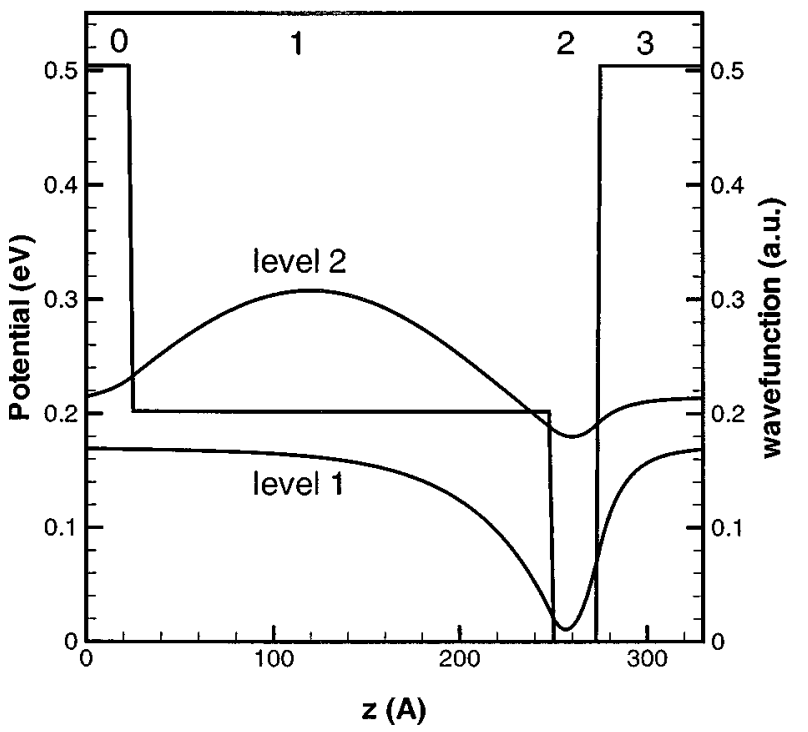

FIG. 8. Quantum well potential profile of structure 1(a) with electron wave functions associated with the first two levels. Layer 1, 2, 3 and 4 are $\mathrm{Al}_{0.6} \mathrm{Ga}_{0.4} \mathrm{As}, \mathrm{Al}_{0.25} \mathrm{Ga}_{0.75} \mathrm{As}, \mathrm{GaAs}$, and $\mathrm{Al}_{0.6} \mathrm{Ga}_{0.4} \mathrm{As}$, respectively.

in Sec. II, the materials are chosen to be GaAs (material 1), $\mathrm{Al}_{0.25} \mathrm{Ga}_{0.75} \mathrm{As}$ (material 2), and $\mathrm{Al}_{0.6} \mathrm{Ga}_{0.4} \mathrm{As}$ (material 3), respectively.

From Eqs. (24) and (25), with the electron-phonon interaction Hamiltonians written in a general form

$$
H_{e-\mathrm{ph}}=\sum_{q} \frac{1}{\sqrt{A}} e^{i \mathbf{q} \cdot \rho} h(q, z)\left(a_{-\mathbf{q}}^{\dagger}+a_{-\mathbf{q}}\right),
$$

where $h(q, z)=-e \phi(q, z)$. The emission rate is given by

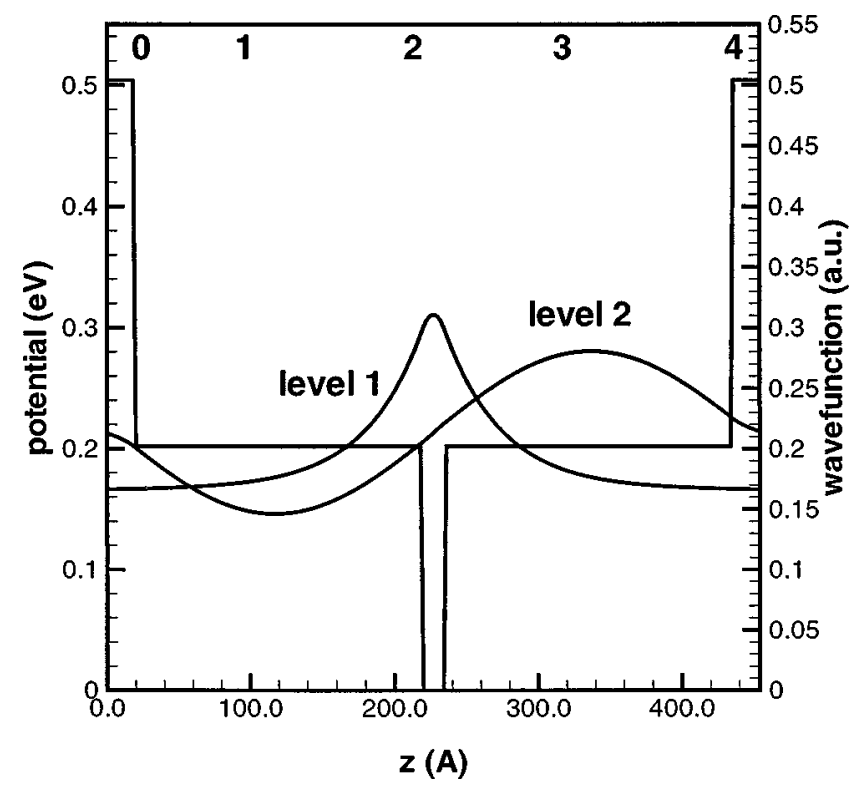

FIG. 9. Quantum well potential profile of structure 1(b) with electron wave functions associated with the first two levels. Layers 1, 2, and 3 are GaAs, $\mathrm{Al}_{0.25} \mathrm{Ga}_{0.75} \mathrm{As}$, and $\mathrm{Al}_{0.6} \mathrm{Ga}_{0.4} \mathrm{As}$, respectively. 


$$
\begin{aligned}
W_{n, n^{\prime}}(\mathbf{k}, \mathbf{q})= & \frac{2 \pi}{\hbar}\left(N_{q}+1\right)|F(q)|^{2} \delta\left(E_{n^{\prime}}+E_{\mathbf{k}-\mathbf{q}}\right. \\
& \left.+\hbar \omega-E_{n}-E_{k}\right),
\end{aligned}
$$

with $F(q)=\int d z h(q, z) \psi_{n}^{*}(z) \psi_{n^{\prime}}(z)$ and the twodimensional in-plane wave vector conservation relation $\mathbf{k}$ $=\mathbf{k}^{\prime}+\mathbf{q}$.

Summing over all allowable final states (or, in other words, summing over q), we calculate the emission rate of an electron in an initial state $|n, \mathbf{k}\rangle$ to a lower subband $n^{\prime}$

$$
W_{n, n^{\prime}}(\mathbf{k})=\sum_{\mathbf{q}} W_{n, n^{\prime}}(\mathbf{k}, \mathbf{q}) .
$$

As discussed above, due to energy conservation, some transition is forbidden. For the emission process, when $E_{\mathbf{k}}$ $<\hbar \omega^{\prime} \equiv \hbar \omega-\left(E_{n}-E_{n^{\prime}}\right)$ and $\omega^{\prime}>0$, i.e., the two subband edges are closer than a phonon energy and the electron does not posses enough initial kinetic energy, there are no final states available and the process is forbidden. For $\omega^{\prime}>0$ and $E_{\mathbf{k}}>\hbar \omega^{\prime}$,

$$
\begin{aligned}
W_{n, n^{\prime}}(\mathbf{k})= & \frac{A}{4 \pi^{2}} \frac{2 \pi}{\hbar}\left(N_{q}+1\right) \int_{-\theta_{\max }}^{\theta_{\max }} d \theta \frac{2 m}{\hbar^{2}} \\
& \times \frac{\left|F\left(q_{+}\right)\right|^{2} q_{+}+\left|F\left(q_{-}\right)\right|^{2} q_{-}}{\left|q_{+}-q_{-}\right|},
\end{aligned}
$$

where

$$
\begin{aligned}
& q_{ \pm}=k \cos \theta \pm \sqrt{k^{2} \cos ^{2} \theta-\frac{2 m}{\hbar} \omega^{\prime},} \\
& \theta_{\max }=\cos ^{-1}\left(\sqrt{\frac{2 m \omega^{\prime}}{\hbar k^{2}}}\right) .
\end{aligned}
$$

For $\omega^{\prime}<0$,

$$
W_{n, n^{\prime}}(\mathbf{k})=\frac{A}{4 \pi^{2}} \frac{2 \pi}{\hbar}\left(N_{q}+1\right) \int_{0}^{2 \pi} d \theta \frac{2 m}{\hbar^{2}} \frac{\left|F\left(q_{+}\right)\right|^{2} q_{+}}{q_{+}} .
$$

The above argument also holds for the absorption process, as long as changes are made for $\omega \rightarrow-\omega, \omega^{\prime} \rightarrow-\omega^{\prime}$, $N_{q}+1 \rightarrow N_{q}$, and $\mathbf{q} \rightarrow-\mathbf{q}$. That is, for the absorption process,

$W_{n, n^{\prime}}(\mathbf{k}, \mathbf{q})=\frac{2 \pi}{\hbar} N_{q}|F(q)|^{2} \delta\left(E_{n^{\prime}}+E_{\mathbf{k}+\mathbf{q}^{-}}-\hbar \omega-E_{n}-E_{k}\right)$,

when $E_{\mathbf{k}}<-\hbar \omega^{\prime}$ and $\hbar \omega^{\prime} \equiv \hbar \omega-\left(E_{n^{\prime}}-E_{n}\right)<0$, i.e., the two subband edges are farther away than a phonon energy and the electron does not have enough initial in-plane energy, there are no final states available and the absorption process is forbidden. For $\omega^{\prime}<0$ and $E_{\mathbf{k}}>-\hbar \omega^{\prime}$,

$$
\begin{aligned}
W_{n, n^{\prime}}(\mathbf{k})= & \frac{A}{4 \pi^{2}} \frac{2 \pi}{\hbar} N_{q} \int_{-\theta_{\max }}^{\theta_{\max }} d \theta \frac{2 m}{\hbar^{2}} \\
& \times \frac{\left|F\left(q_{+}\right)\right|^{2} q_{+}+\left|F\left(q_{-}\right)\right|^{2} q_{-}}{\left|q_{+}-q_{-}\right|},
\end{aligned}
$$

where

$$
\begin{aligned}
& q_{ \pm}=k \cos \theta \pm \sqrt{k^{2} \cos ^{2} \theta+\frac{2 m}{m} \omega^{\prime},} \\
& \theta_{\max }=\cos ^{-1}\left(\sqrt{-\frac{2 m \omega^{\prime}}{\hbar k^{2}}}\right) .
\end{aligned}
$$

For $\omega^{\prime}>0$,

$$
W_{n, n^{\prime}}(\mathbf{k})=\frac{A}{4 \pi^{2}} \frac{2 \pi}{\hbar} N_{q} \int_{0}^{2 \pi} d \theta \frac{2 m}{\hbar^{2}} \frac{\left|F\left(q_{+}\right)\right|^{2} q_{+}}{q_{+}} .
$$

To proceed further we need the electronic wave functions. Instead of assuming some form of the wave functions, ${ }^{12}$ we seek numerical solutions as they enable us to examine various structures, such as the asymmetric and symmetric structures as shown in Figs. 8 and 9.

For the half space modes and confined LO modes, the numerical results obtained from our calculation show that the coupling between electron wave functions and these modes is negligible compared to that between electrons and bulk or interface modes. This is because the first two electronic levels are essentially localized inside the well while half space modes are outside the well, therefore the overlapping integral of $F(q)$ is minimal. For the confined modes with $m$ $=1,3,5, \cdots$, since we are considering two consecutive electronic subbands which always have opposite parity in the symmetric structure or near-opposite parity in the asymmetric case, these modes have zero or near-zero coupling with neighboring electronic subbands due to the symmetric polarization of these modes. For the confined LO modes with $m$ $=2,4,6, \cdots$, the coupling is larger but still negligible as compared to the electron-bulk-mode and electron-interfacemode coupling.

For the interface modes, typical comparisons between transition rates due to the bulk mode and the chosen interface mode are shown in Fig. 10 (for the asymmetric structure in Fig. 8) and Fig. 11 (for the symmetric structure in Fig. 9), respectively. The emission rates due to electron interaction with the interface modes are about one order of magnitude larger than those calculated using the bulk Fröhlich Hamiltonian. We found that, for certain interface modes, the ratio of the emission rates due to the interface mode scattering and the rate calculated using the bulk Fröhlich Hamiltonian can be as large as 50. Carefully examining these interface phonon modes, we found that these modes usually have one to two orders of magnitude larger amplitude than the other interface modes. In Fig. 10, for the asymmetric structure, the rate obtained using the bulk Fröhlich Hamiltonian is compared to the rate of an interface phonon emission with the phonon energy near $34.8 \mathrm{meV}$, which is close to the subband separation $E_{2}-E_{1}=33.7 \mathrm{meV}$. In Fig. 11, the same comparison is made for the symmetric structure, showing an emission rate assisted by an antisymmetric interface mode of $50 \mathrm{meV}$, with $E_{2}-E_{1}=47.1 \mathrm{meV}$. An antisymmetric mode is shown here, while the symmetric modes have near-zero coupling to the first two levels due to the parity consideration. In these two figures, the emission rates due to the half space modes and confined phonon modes are all negligible and merge into the $x$ axis. These results indicate that the interface phonon-assisted transition rates can be one to two 


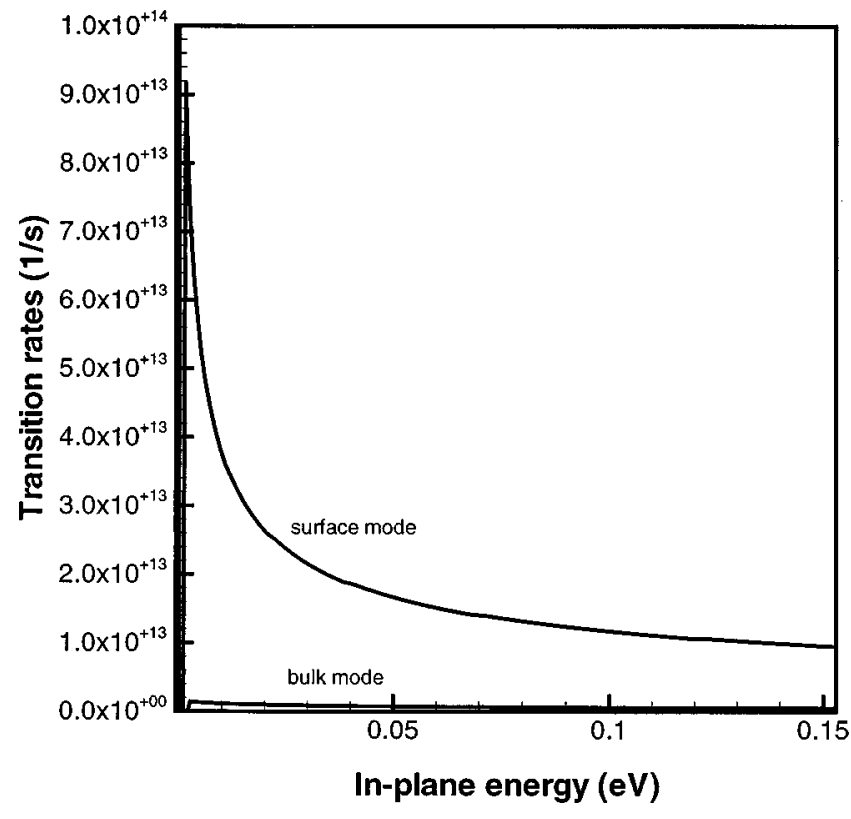

FIG. 10. Comparison of the transition rates for the asymmetric structure. Only the contribution of one surface mode of $34.8 \mathrm{meV}$ is shown. The energy level separation is $33.7 \mathrm{meV}$ for this plot. The maximum rate due to the surface mode is about 50 times larger than that of the bulk mode. The rates for the confined and half space modes are negligible and indiscernible from the $x$ axis.

orders of magnitude larger than those calculated with the bulk Fröhlich Hamiltonian, which agrees with the results of Ref. 12. In Figs. 12 and 13, the maxima of emission rates (the maxima of $W \mathrm{~s}$ as functions of $E_{\mathbf{k}}$ ) as functions of the level separation $E_{2}-E_{1}$ for different phonon modes are plotted, and those of the interface mode and bulk mode are compared. Figure 12 shows the maximum rates calculated using

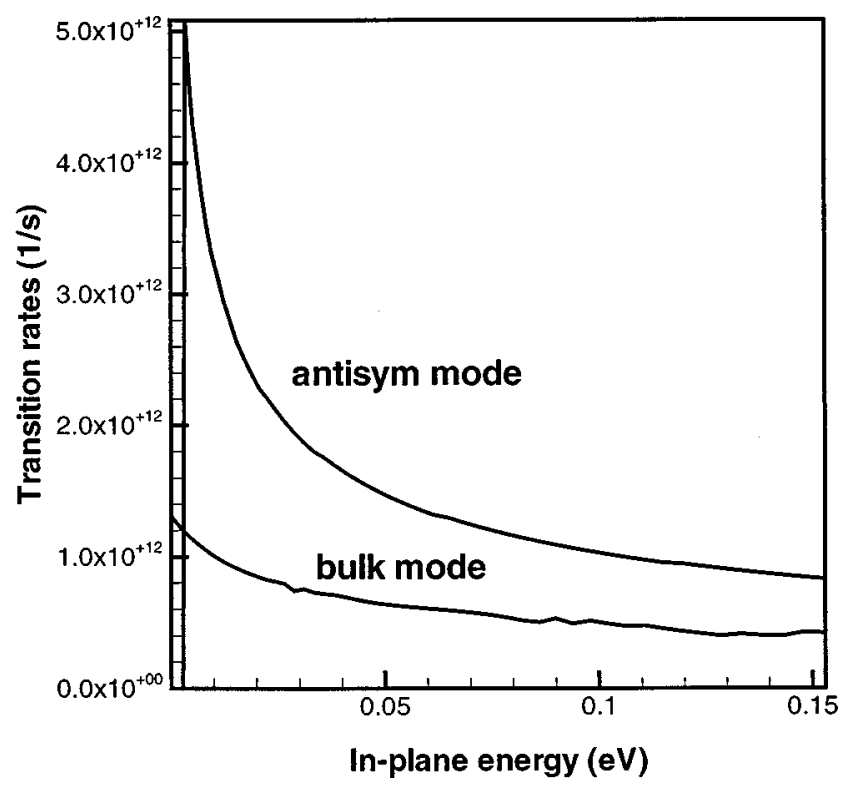

FIG. 11. Comparison of the transition rates for the symmetric structure. Only the contribution of one antisymmetric interface mode of $50 \mathrm{meV}$ is shown. The rates for the confined and half space modes are negligible and indiscernible from the $x$ axis. The rates for the symmetric modes are zero due to the parity consideration.

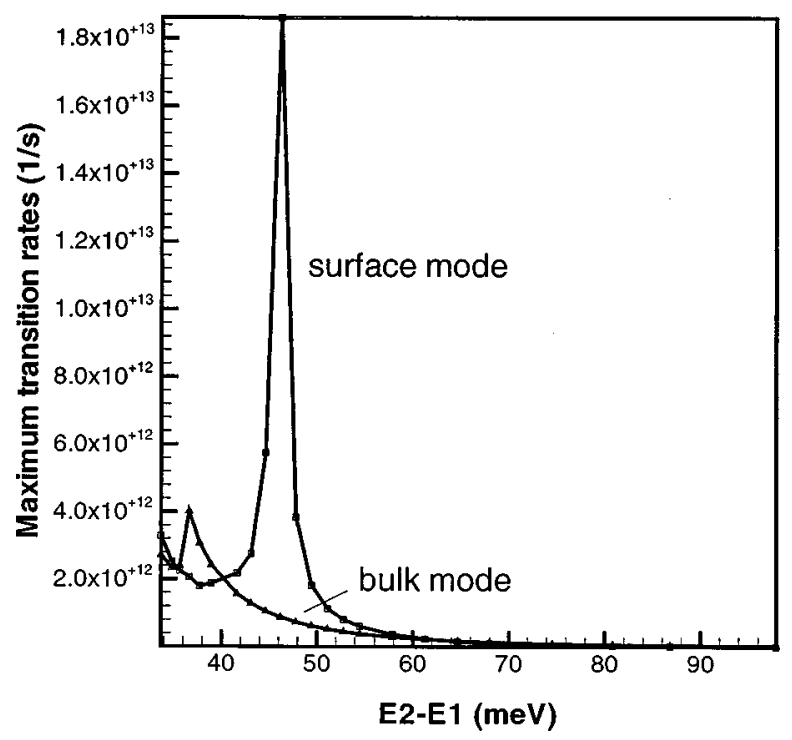

FIG. 12. Comparison of the peak transition rates as functions of level separation for the asymmetric structure. The surface mode has an energy of 48 $\mathrm{meV}$.

the bulk mode and an interface mode with energy near 48 meV for the asymmetric structure. In Fig. 13, the maximum rates calculated using the bulk mode and an interface mode with energy near $50 \mathrm{meV}$ for the symmetric structure are compared. These results confirm that the interface phononassisted transition rates are significantly larger than those obtained from the bulk mode interaction Hamiltonian.

The form factors can be defined through the effective electron-electron interactions mediated by phonons. The effective electron-electron interaction due to exchange of phonon mode $s$ can be formally written as ${ }^{9}$

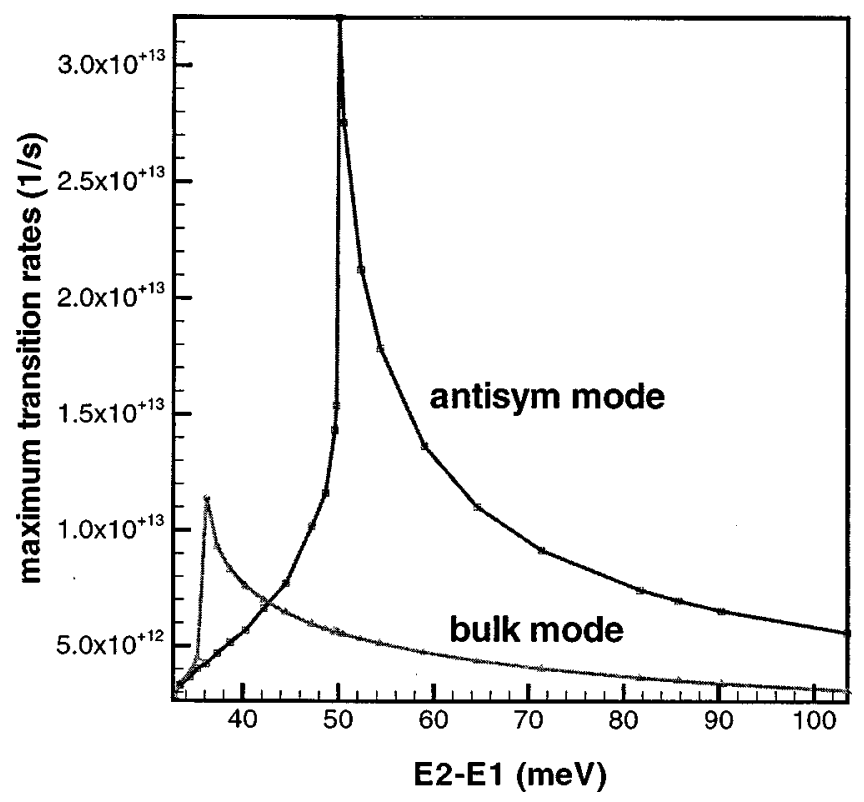

FIG. 13. Comparison of the peak transition rates as functions of level separation for the symmetric structure. The surface mode has an energy of 50 $\mathrm{meV}$. 


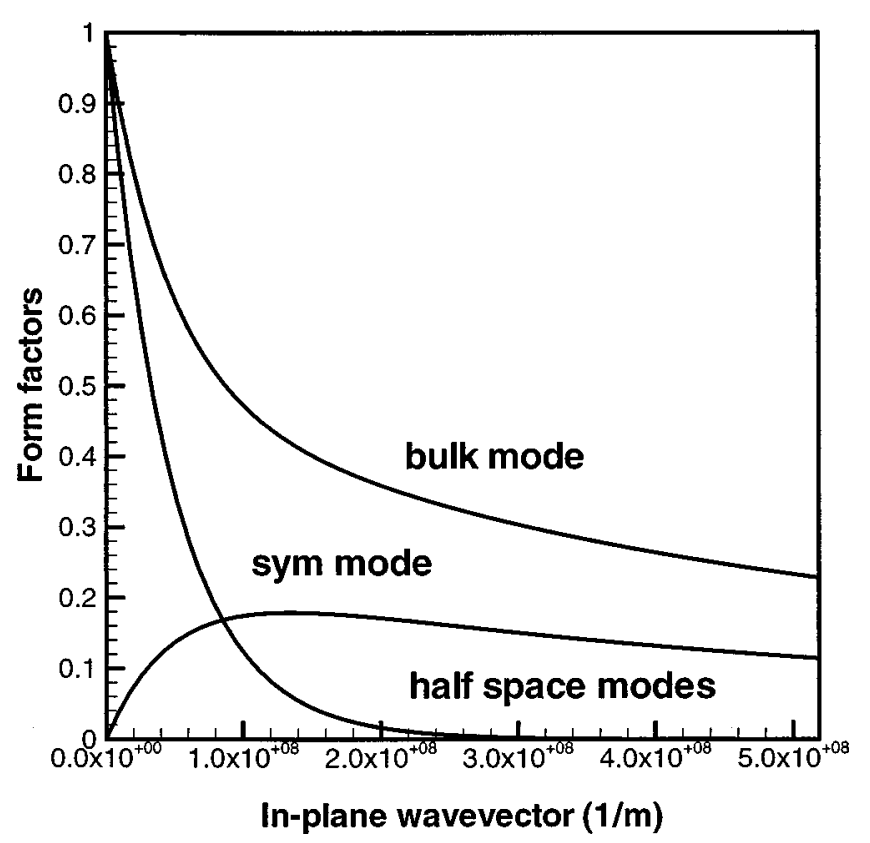

FIG. 14. The form factors of various phonon modes in the symmetric structure. The electron wave function of the second level is used. The form factors of the confined LO mode and antisymmetric mode are negligible compared to those for the symmetric and half space modes.

$$
H_{e-e}(q, i \nu)=2 \pi \alpha_{s}\left(\frac{2 \omega_{s}^{3}}{m^{*}}\right)^{1 / 2} \frac{1}{2 q} F_{s}(q) D_{s}(i \nu),
$$

where $\alpha_{j}$ is the coupling constant defined as the term independent of the electron wave functions and it reduces to the bulk Fröhlich coupling constant when the differences among materials are neglected, $D_{s}(i \nu)=2 \omega_{s} /\left[(i \nu)^{2}-\omega_{s}^{2}\right]$ is the phonon Green's function, $\omega_{s}$ is the frequency of phonon mode $s, m^{*}$ is the electron effective mass, and the form factor $F_{s}(q)$ is defined as the term independent of material parameters. Since the form factors depend only on the electron wave functions associated with the quantized $z$ motion and phonon electrostatic potential profiles, the sum of the form factors for various phonon modes should be equal to the form factor for bulk modes, due to the orthonormality of the phonon eigenmodes. Therefore, if phonon eigenfrequencies and the coupling constants of the interaction Hamiltonians were the same as those of bulk phonons, the sum of transition rates induced by all phonon modes in a quantum well structure would just be equal to the rate by bulk phonons. However, for interface modes the transition rates are sensitive functions of eigenfrequencies, possibly having orders of magnitude difference among transition rates by the interface modes of different frequencies. Nonetheless, the sum rule provides a way to examine the phonon mode calculations. The form factors for the calculated phonon modes in a simple double heterostructure are shown in Fig. 14, where the electron wave function of the second level in the structure is used. In this specific case, the form factors for the confined LO mode and antisymmetric interface mode are negligible as compared to those for the symmetric interface and half space modes. The sum rule is well observed in our calculation.

\section{CONCLUSION}

Optical phonon modes and their effects on electron relaxation rates in the step quantum well structures are studied in this work. The various phonon modes, including the interface phonon modes, confined LO modes, and half space modes are calculated using the transfer matrix method, based on the dielectric continuum model. We have examined and compared electron transition rates obtained using the bulk Fröhlich Hamiltonian and interaction Hamiltonian with the localized phonon modes. It is concluded that, in narrow quantum well structures, instead of employing the simple electron bulk-phonon interaction, the interface phonon modes have to be considered as they play a very important role in phonon-assisted intersubband transitions.

\section{ACKNOWLEDGMENT}

This work was supported, in part, by the U.S. Army Research Office, Contract No. DAAL03-91-C-0034.

${ }^{1}$ J. Faist, F. Capasso, D. L. Sivco, C. Sirtori, A. L. Hutchinson, and A. Y. Cho, Science 264, 553 (1994).

${ }^{2}$ X. Zhang, G. I. Haddad, J. P. Sun, A. Afzali-Kushaa, C. Y. Sung, and T. Norris, in 1995 53rd Annual Device Research Conference Digest (IEEE, New York, 1995), p. 118

${ }^{3}$ C. Y. Sung, T. B. Norris, A. Afzali-Kushaa, and G. I. Haddad, Appl. Phys. Lett. 68, 435 (1996).

${ }^{4}$ X. Zhang, C. Y. Sung, T. B. Norris, and G. I. Haddad, Proc. SPIE 2694, 19 (1996)

${ }^{5}$ A. A. Lucas, E. Kartheuser, and R. G. Badro, Phys. Rev. B 2, 2488 (1970).

${ }^{6}$ J. J. Licari and R. Evrard, Phys. Rev. B 15, 2254 (1977).

${ }^{7}$ S. Yu, K. W. Kim, M. A. Stroscio, G. J. Iafrate, J. P. Sun, and G. I. Haddad, J. Appl. Phys. 82, 3363 (1997).

${ }^{8}$ R. Fuchs and K. L. Kliewer, Phys. Rev. 140, A2076 (1965); K. L. Kliewer and R. Fuchs, Phys. Rev. 144, 495 (1966).

${ }^{9}$ N. Mori and T. Ando, Phys. Rev. B 40, 6175 (1989).

${ }^{10}$ M. V. Kisin, V. B. Gorfinkel, M. A. Stroscio, G. Belenky, and S. Luryi, J. Appl. Phys. 82, 2031 (1997).

${ }^{11}$ M. V. Kisin, M. A. Stroscio, V. B. Gorfinkel, G. Belenky, and S. Luryi, presented at Conference on Lasers and Electro-Optics, Baltimore, MD, May 18-23, 1997.

${ }^{12}$ M. A. Stroscio, J. Appl. Phys. 80, 6864 (1996).

${ }^{13}$ J. P. Sun, H. B. Teng, G. I. Haddad, M. A. Stroscio, and G. J. Iafrate, presented at Fifth International Workshop on Computational Electronics, Notre Dame, IN, May 28-31 1997 (to be published in VLSI Design).

${ }^{14}$ J. P. Sun, H. B. Teng, G. I. Haddad, and M. A. Stroscio, presented at 2nd International Workshop on Surfaces and Interfaces in Mesoscopic Devices, Maui, Hawaii, December 8-12, 1997 (to be published in Semicond. Sci. Technol).

${ }^{15}$ N. Bannov, V. Mitin, and M. A. Stroscio, in Proceedings of the International Device Research Symposium, University of Virginia, December 1993, pp. 659-662.

${ }^{16}$ K. W. Kim and M. A. Stroscio, J. Appl. Phys. 68, 6289 (1990). 